\title{
Searches for direct pair production of third generation squarks with the ATLAS detector
}

\author{
Jovan Mitrevski* \\ Fakultät für Physik, Ludwig-Maximilians-Universität München, Germany \\ E-mail: Jovan.Mitrevski@cern.ch
}

\begin{abstract}
Naturalness arguments for weak-scale supersymmetry favor supersymmetric partners of the third generation quarks with masses not too far from those of their Standard Model counterparts. Top or bottom squarks with masses less than or around one $\mathrm{TeV}$ can also give rise to direct pair production rates at the LHC that can be observed in the data sample recorded by the ATLAS detector. The paper presents recent ATLAS results from searches for direct stop and sbottom pair production, using the data collected during the LHC Run 2.
\end{abstract}

XXVI International Workshop on Deep-Inelastic Scattering and Related Subjects (DIS2018) 16-20 April 2018

Kobe, Japan

\footnotetext{
* Speaker, on behalf of the ATLAS Collaboration.
} 
Supersymmetry (SUSY) proposes a symmetry between fermions and bosons, which in the Minimal Supersymmetric Standard Model (MSSM) adds partners to the Standard Model (SM) particles that differ by $1 / 2$ in spin but with otherwise identical quantum numbers. One of the motivations for SUSY is that it stabilizes the Higgs mass. Higher-order corrections that would causes divergences in the SM without fine-tuning instead cancel with SUSY. However, SUSY is not a perfect symmetry or else the SUSY partners to the SM particles would have identical mass with the SM particles. With SUSY being a broken symmetry, fine-tuning can still be kept small if the SUSY masses are not much greater than the electroweak scale.

Given the sizes of the Yukawa couplings, the third generation quarks and their SUSY partners, know as the stop $(\tilde{t})$ and sbottom $(\tilde{b})$, introduce the most fine-tuning. Limiting fine-turning therefore requires the masses of the third generation squarks to not be too high. Additionally, renormalization group equations often drive these masses down. Thus, searching for direct stop and sbottom production is theoretically well motivated.

This paper presents searches for direct stop and sbottom pair production using $36.1 \mathrm{fb}^{-1}$ of data collected by the ATLAS detector in 2015 and 2016. All the analyses assume $R$-parity is conserved, resulting in sparticles being pair-produced and a stable lightest SUSY particle (LSP). In all the analyses except one, the LSP is assumed to be a neutralino $\left(\tilde{\chi}^{0}\right)$, the neutral supersymmetric partner to a gauge or Higgs boson.

The ATLAS detector [1] has a symmetric cylindrical geometry and nearly $4 \pi$ solid angle coverage. Closest to the beam-line is the inner detector (ID), consisting of pixel, microstrip, and transition radiation trackers inside a $2 \mathrm{~T}$ magnetic field. Next is a presampler followed by a lead/liquidargon (LAr) electromagnetic (EM) calorimeter in the region $|\eta|<3.2$. Iron/scintillating-tile or LAr hadronic calorimeters are located behind the EM calorimeter. LAr calorimeters for both EM and hadronic energy are located in the forward region. A muon spectrometer (MS) with a toroidal magnet is located outside the calorimeters.

The analyses use jets reconstructed using an anti- $k_{\mathrm{T}}$ algorithm with a radius parameter of 0.4 [2]. These jets are sometimes "reclustered" into larger jets of radius parameter 1.2 or 0.8 to capture boosted objects. A multivariate algorithm is used for $b$-tagging jets. Electrons are reconstructed using clusters in the EM calorimeter matched to tracks. Signal muons are reconstructed from tracks combining ID and MS hits or from MS tracks compatible with coming from the collision in the region outside the ID acceptance. Hadronically-decaying taus are constructed from jets and one or three tracks using a multivariate technique. The $\mathbf{E}_{\mathrm{T}}^{\text {miss }}$ is calculated from baseline electrons, muons, jets, and a soft term built from preselected tracks associated with the primary vertex. Events that contain jets likely coming from non-collision sources or detector noise or muons that are badly reconstructed or likely coming from cosmic sources are removed from the analyses.

The transverse mass $\left(m_{\mathrm{T}}\right)$, which has an end-point when there is one unreconstructed particle, and the generalized form for when there are two unreconstructed particles, the stransverse mass $\left(m_{\mathrm{T} 2}\right)$, are used by the analyses [3]. Top reconstruction is widely used to discriminate against (or select) $t \bar{t}$ events. In particular, a $\chi^{2}$ minimization is used to select hadronic tops decaying into three jets. Alternatively, jet reclustering, starting with a radius parameter 3.0 anti- $k_{\mathrm{T}}$ jet and shrinking the size, is sometimes used to select top and $W$ jets. For events that do not produce much $E_{\mathrm{T}}^{\text {miss }}$, a selection using boosting from an ISR jet can be useful. In particular, the Recursive Jigsaw Reconstruction (RJR) technique [4] or the related super-razor variables [5] are often used. 




Figure 1: Models with neutralino LSP considered by the analyses [6].
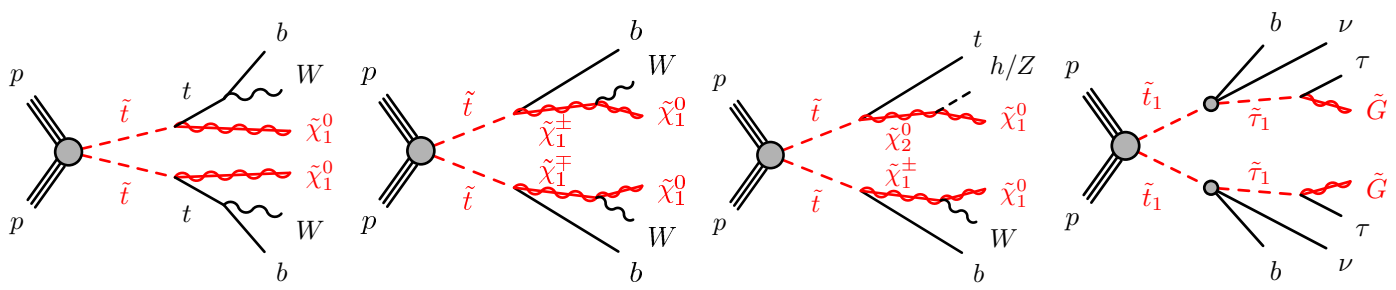

Figure 2: Feynman diagrams for the direct production of stop pairs and their decays. The left diagram shows only $\tilde{t}_{1} \rightarrow t \tilde{\chi}_{1}^{0}$ decays (with a potentially off-shell $t$ ), while the two middle diagrams show other possible decays if $\tilde{\chi}_{1}^{ \pm}$or $\tilde{\chi}_{2}^{0}$ are kinematically accessible [7]. The right diagram shows decays in a GMSB scenario with a stau NLSP [8].

The right and left handed stops $\left(\tilde{t}_{\mathrm{R}}\right.$ and $\tilde{t}_{\mathrm{L}}$ ) mix, forming $\tilde{t}_{1}$ and $\tilde{t}_{2}$, with $\tilde{t}_{1}$ being the lighter, and similarly the left and right handed sbottoms $\left(\tilde{b}_{\mathrm{R}}\right.$ and $\left.\tilde{b}_{\mathrm{L}}\right)$ mix, forming $\tilde{b}_{1}$ and $\tilde{b}_{2}$, with $\tilde{b}_{1}$ being the lighter. If $\tilde{t}_{1}$ is made up mostly of $\tilde{t}_{\mathrm{L}}$, then $\tilde{b}_{1}$ is usually of similar mass. The neutralinos and charginos also separately mix, with the subscripts ordering them by mass.

Figure 1 shows some models considered by these analyses. The simplest model, case (a), only has a bino-like neutralino (the SUSY partner of a $B$ boson) LSP with a mass lower than $\tilde{t}_{1}$, resulting in production and decay like the left diagram in Figure 2. In the analyses summarized here, $\tilde{t}_{1}$ is assumed to be mostly $\tilde{t}_{\mathrm{R}}$. If $\Delta m\left(\tilde{t}_{1}, \tilde{\chi}_{1}^{0}\right)>m_{t}$, we have an on-shell top quark in the decay, $\tilde{t}_{1} \rightarrow t \tilde{\chi}_{1}^{0}$, resulting in a two-body decay. If $m_{b}+m_{W}<\Delta m\left(\tilde{t}_{1}, \tilde{\chi}_{1}^{0}\right)<m_{t}$, the top quark in the decay is off-shell, resulting in the three-body decay, $\tilde{t}_{1} \rightarrow b W \tilde{\chi}_{1}^{0}$. If $\Delta m\left(\tilde{t}_{1}, \tilde{\chi}_{1}^{0}\right)$ is even smaller, then the $W$ boson would also be off-shell, resulting in the four-body decay, $\tilde{t}_{1} \rightarrow b f f^{\prime} \tilde{\chi}_{1}^{0}$. Alternately, we may have the flavor-changing neutral current decay, $\tilde{t}_{1} \rightarrow c \tilde{\chi}_{1}^{0}$.

Case (b) in Figure 1 shows the "wino NLSP" case, where wino-like $\tilde{\chi}_{1}^{ \pm}$and $\tilde{\chi}_{2}^{0}$ (SUSY partners to the $W$ boson) have masses between the bino-like LSP and the $\tilde{t}_{1}$. Gauge unification generally predicts the wino to be about twice as heavy as the bino, so the analyses set limits in that scenario, though they also explore the case where the $\tilde{t}_{1}$ is close to the wino mass, which may result in $b$ tagging being inefficient due to the softness of the $b$-jets. In these analyses $\tilde{t}_{1}$ is considered to be mainly $\tilde{t}_{\mathrm{L}}$, meaning $m_{\tilde{b}_{1}} \approx m_{\tilde{t}_{1}}$, and direct $\tilde{b}_{1}$ pairs are also produced. The production and decays in this case can additionally proceed as the two middle diagrams in Figure 2. Those generally dominate unless they are kinematically suppressed. The $\tilde{\chi}_{2}^{0}$ decay depends significantly on the sign 
of $\mu$, decaying mostly to $h \tilde{\chi}_{1}^{0}$ if positive, $Z \tilde{\chi}_{1}^{0}$ if negative. Sbottom pairs, if produced, tend to decay analogously with $\tilde{b}_{1} \rightarrow t \tilde{\chi}_{1}^{ \pm}$and $\tilde{b}_{1} \rightarrow b \tilde{\chi}_{2}^{0}$ decays.

Case (c) shows the case where the LSP is a higgsino-like neutralino. Such models are usually motivated by naturalness since the higgsino must be light to avoid fine-tuning. As in the wino NLSP case, the left three diagrams in Figure 2 are allowed; however, $\Delta m\left(\tilde{\chi}_{1}^{ \pm}, \tilde{\chi}_{1}^{0}\right)$ and $\Delta m\left(\tilde{\chi}_{2}^{0}, \tilde{\chi}_{1}^{0}\right)$ are small (few GeV), so $W$ and $Z$ bosons in the decays are off-shell, resulting in soft leptons in analyses looking for leptons. The branching ratios depend on the relative fraction of $\tilde{t}_{\mathrm{R}}$ and $\tilde{t}_{\mathrm{L}}$ in $\tilde{t}_{1}$. In the simplified models used by the analyses, only $\tilde{t}_{1}$ production is considered.

Case (d) shows the case where the LSP is a mixture of higgsino and bino, which can provide a viable dark matter candidate [9]. Phenomenologically, these models have a longer decay chain than the others. Both $\tilde{t}_{1}$ and $\tilde{b}_{1}$ production is considered where applicable.

The analyses define various signal regions (SRs) where to look for a signal, control regions (CRs) where to measure the background, and validation regions (VRs) to validate the background modeling. One analysis searches for stop pair production with no electrons of muons in the decay [7], using an $E_{\mathrm{T}}^{\text {miss }}$ trigger and requiring $E_{\mathrm{T}}^{\text {miss }}>250 \mathrm{GeV}$ and four jets. At least one of the jets needs to be $b$-tagged in all cases, though some signal regions require more. Two SRs are defined to look for high-mass $\tilde{t}_{1}$ with two-body $\tilde{t}_{1} \rightarrow t \tilde{\chi}_{1}^{0}$ decays using reclustered jets. Two reclustered jets are required, either both having a mass above $120 \mathrm{GeV}$, one having a mass above $120 \mathrm{GeV}$ and one between 60 and $120 \mathrm{GeV}$, or one having a mass above $120 \mathrm{GeV}$ and one below $60 \mathrm{GeV}$. The different cases are statistically combined to maximize sensitivity. Another SR uses RJR to target the region where $\Delta m\left(\tilde{t}_{1}, \tilde{\chi}_{1}^{0}\right) \approx m_{t}$. The SR is divided into different ISR boost windows, which are statistically combined. A SR (split into two orthogonal subregions) is defined targeting stop production with $\tilde{t}_{1} \rightarrow b \tilde{\chi}_{1}^{ \pm}$decays, and finally a SR is defined targeting highly boosted jets.

An analysis selecting decays with one lepton (electron or muon) [6] has three main configurations: $E_{\mathrm{T}}^{\text {miss }}$ trigger with high lepton $p_{\mathrm{T}}$ for SRs that use a high $E_{\mathrm{T}}^{\text {miss }}$ selection; lepton trigger with high lepton $p_{\mathrm{T}}$, for SRs that have a lower $E_{\mathrm{T}}^{\text {miss }}$ selection (at the expense of tighter lepton requirements); and $E_{\mathrm{T}}^{\text {miss }}$ trigger with soft leptons, for analyses that use electrons (muons) with $p_{\mathrm{T}}>5(4) \mathrm{GeV}$ targeting off-shell gauge bosons. There are two SRs targeting the bino LSP scenario with a two-body decay in the bulk, one of which included a shape fit in $E_{\mathrm{T}}^{\text {miss }}$. There are three SRs targeting different $\tilde{t}_{1}$ masses along the $\Delta m\left(\tilde{t}_{1}, \tilde{\chi}_{1}^{0}\right) \approx m_{t}$ diagonal, all of which use boosted decision trees (BDTs), two of them making a shape fit on the BDT score. The 3-body and 4-body scenarios have their own SRs defined, with the latter using soft leptons. Three SRs target the wino NLSP model, including one with a $b$-veto for when $\Delta m\left(\tilde{t}_{1}, \tilde{\chi}_{1}^{ \pm}\right)$is small. Three soft lepton SRs target the higgsino grid.

An analysis selecting decays with two leptons [10] define two-body SRs targeting $\tilde{t}_{1} \rightarrow t \tilde{\chi}_{1}^{0}$ and $\tilde{t}_{1} \rightarrow b \tilde{\chi}_{1}^{ \pm}$decays and three-body SRs separately targeting $\Delta m\left(\tilde{t}_{1}, \tilde{\chi}_{1}^{0}\right) \approx m_{t}$ and $\Delta m\left(\tilde{t}_{1}, \tilde{\chi}_{1}^{0}\right) \approx m_{W}$ using super-razor variables. Those signal regions use a di-lepton trigger. Additionally, soft-lepton SRs are defined targeting the four-body decays, using $E_{\mathrm{T}}^{\text {miss }}$ triggers.

No significant excess was observed in the above analyses. Figure 3 summarizes the exclusion limits in the four models discussed above.

Another analysis looks for cases with $Z$ or $h$ bosons in the decay [12], specifically looking for leptonic $Z$ decays or $h \rightarrow b \bar{b}$ decays. Additionally, a $Z$ or $h$ boson can appear in $\tilde{t}_{2} \rightarrow Z \tilde{t}_{1}$ or $\tilde{t}_{2} \rightarrow h \tilde{t}_{1}$ decays, which may be important if the $\tilde{t}_{1}$ is too difficult to be directly detected. Three 

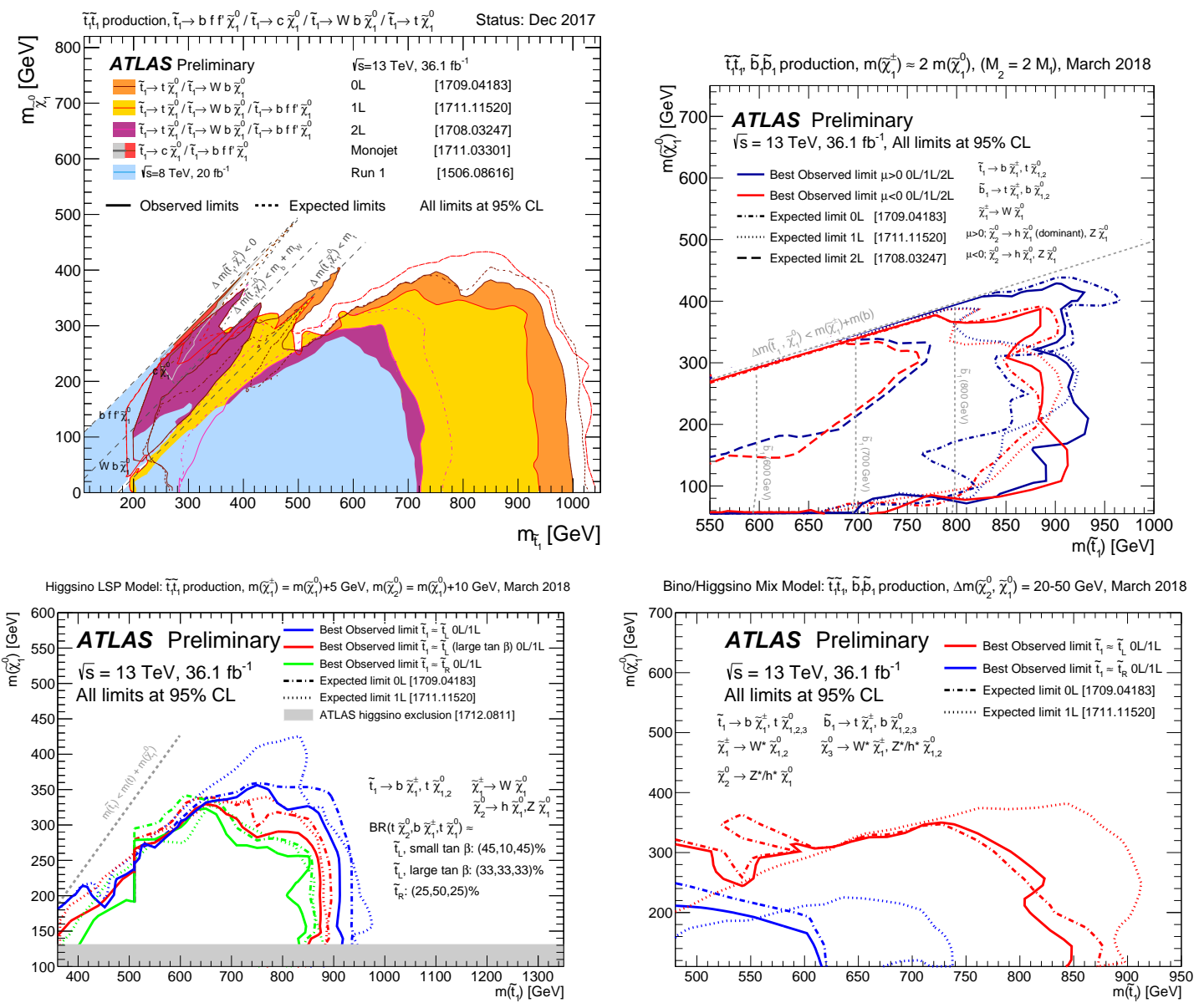

Figure 3: Exclusion limits for direct production of stop pairs in the bino LSP (upper left), wino NLSP (upper right) models, higgsino LSP (lower left), and bino-higgsino LSP (lower right) models [11].

SRs are defined requiring at least three leptons and one $b$-tagged jet targeting events with $Z$ bosons, and three SRs are defined with 1-2 leptons and at least four $b$-tagged jets targeting events with $h$ bosons. Since the two categories of SRs are orthogonal, the best expected SRs from each category are statistically combined. No significant excess was observed. The results exclude $\tilde{t}_{2}$ and $\tilde{t}_{1}$ masses up to about $800 \mathrm{GeV}$.

One analysis looks for top or charm squark pair production with charm-tagged jets in the decay [13]. The branching ratio of $\tilde{t}_{1} \rightarrow c \tilde{\chi}_{1}^{0}$ can exceed three- and four-body decays in certain scenarios. Assuming a $100 \%$ branching ratio to $c \tilde{\chi}_{1}^{0}$, top and charm squarks with masses up to $850 \mathrm{GeV}$ are excluded for a massless $\tilde{\chi}_{1}^{0}$, and masses up to $500 \mathrm{GeV}$ are excluded when the squark to $\tilde{\chi}_{1}^{0}$ mass difference is less than $100 \mathrm{GeV}$.

One analysis, focusing on direct $\tilde{b}_{1}$ production [14], selects events with two or more jets (depend on SR), at least two $b$-tagged. A zero lepton selection targets $\tilde{b}_{1} \rightarrow b \tilde{\chi}_{1}^{0}$ decays, while a one lepton selection additionally targets $\tilde{b}_{1} \rightarrow t \tilde{\chi}_{1}^{ \pm}, \tilde{t}_{1} \rightarrow t \tilde{\chi}_{1}^{0}$, and $\tilde{t}_{1} \rightarrow b \tilde{\chi}_{1}^{ \pm}$, with $\Delta m\left(\tilde{\chi}_{1}^{ \pm}, \tilde{\chi}_{1}^{0}\right)=1 \mathrm{GeV}$. The selections are statistically combined when appropriate. No significant excess was observed.

The last analysis summarized in this paper looks at stop pair production in a GMSB model, 
with a gravitino LSP and the SUSY partner of the tau (stau) as the next to lightest SUSY particle (NLSP) [8]. The process studied is shown in the rightmost diagram of Figure 2. One SR is defined requiring two hadronic tau decays and another requiring one hadronic tau decay and one electron or muon, which is assumed to come from a leptonic tau decay. In a simplified model, masses up to $m_{\tilde{t}_{1}}=1.16 \mathrm{TeV}$ and $m_{\tilde{\tau}_{1}}=1.00 \mathrm{TeV}$ are excluded.

A broad search for direct production of third generation squarks was done by the ATLAS experiment at the LHC with no significant excess found. The analyses are currently being optimized and the methods improved for an update with the full Run 2 dataset.

\section{References}

[1] ATLAS Collaboration, The ATLAS Experiment at the CERN Large Hadron Collider, JINST 3 (2008) S08003.

[2] M. Cacciari, G. P. Salam and G. Soyez, The Anti- $k_{\mathrm{T}}$ jet clustering algorithm, JHEP 04 (2008) 063 [arXiv:0802.1189].

[3] C. G. Lester and D. J. Summers, Measuring masses of semiinvisibly decaying particles pair produced at hadron colliders, Phys. Lett. B463 (1999) 99 [hep-ph/ 9906349 ].

[4] P. Jackson, C. Rogan and M. Santoni, Sparticles in motion: Analyzing compressed SUSY scenarios with a new method of event reconstruction, Phys. Rev. D95 (2017) 035031 [arXiv: 1607.08307 ].

[5] M. R. Buckley, J. D. Lykken, C. Rogan and M. Spiropulu, Super-Razor and Searches for Sleptons and Charginos at the LHC, Phys. Rev. D89 (2014) 055020 [arXiv: 1310 . 4827].

[6] ATLAS Collaboration, Search for top-squark pair production in final states with one lepton, jets, and missing transverse momentum using $36 \mathrm{fb}^{-1}$ of $\sqrt{s}=13 \mathrm{TeV}$ pp collision data with the ATLAS detector, arXiv:1711.11520.

[7] ATLAS Collaboration, Search for a scalar partner of the top quark in the jets plus missing transverse momentum final state at $\sqrt{s}=13 \mathrm{TeV}$ with the ATLAS detector, JHEP 12 (2017) 085 [arXiv:1709.04183].

[8] ATLAS Collaboration, Search for top squarks decaying to tau sleptons in pp collisions at $\sqrt{s}=13$ TeV with the ATLAS detector, arXiv:1803.10178.

[9] N. Arkani-Hamed, A. Delgado and G. F. Giudice, The Well-tempered neutralino, Nucl. Phys. B741 (2006) 108 [hep-ph/ 0601041$].$

[10] ATLAS Collaboration, Search for direct top squark pair production in final states with two leptons in $\sqrt{s}=13$ TeV pp collisions with the ATLAS detector, Eur. Phys. J. C77 (2017) 898 [arXiv:1708.03247].

[11] https://atlas.web.cern.ch/Atlas/GROUPS/PHYSICS/CombinedSummaryPlots/SUSY/.

[12] ATLAS Collaboration, Search for direct top squark pair production in events with a Higgs or Z boson, and missing transverse momentum in $\sqrt{s}=13 \mathrm{TeV}$ pp collisions with the ATLAS detector, JHEP 08 (2017) 006 [arXiv: 1706.03986$].$

[13] ATLAS Collaboration, Search for supersymmetry in final states with charm jets and missing transverse momentum in $13 \mathrm{TeV}$ pp collisions with the ATLAS detector, arXiv:1805.01649.

[14] ATLAS Collaboration, Search for supersymmetry in events with b-tagged jets and missing transverse momentum in pp collisions at $\sqrt{s}=13 \mathrm{TeV}$ with the ATLAS detector, JHEP 11 (2017) 195 [arXiv:1708.09266]. 